\title{
THE SPECTRAL RADIUS OF A DIRECT INTEGRAL OF OPERATORS
}

\author{
T. R. CHOW ${ }^{1}$
}

ABSTRACT. The purpose of this paper is to give a formula for computing the spectral radius of a direct integral of operators from the numerical radius of its integrand. The direct sum of operators is a special case of our theorem. An example is given where our theorem cannot be applied.

P. Halmos presented in his book [2] an operator with positive spectral radius which is a direct sum of nilpotent operators. The example is a unilateral weighted shift defined by the sequence

$$
1,0,1,1,0,1,1,1,0,1,1,1,1,0, \cdots \text {. }
$$

The misbehavior of the approximate point spectrum in this case represents a certain degree of difficulty and hence discourages people from studying the spectral radius of a direct integral of operators. However, in the above example, the norm, the numerical radius, and the spectral radius are all the same. This suggests that there is still some hope at least for obtaining some partial results.

There are cases in which one can calculate the spectral radius of a direct integral of operators from that of its integrand; however, we shall discuss this elsewhere. The purpose of this paper is to establish a formula for calculating the spectral radius in terms of the numerical radius. In case the direct integral reduces to a direct sum, our theorem, which was suggested to the author by N. Suzuki, will explain the example mentioned at the beginning of this paper. Thanks are due to Professor Suzuki.

By an operator on a (complex) Hilbert space $H$, we shall always mean a bounded linear transformation from $H$ into itself, and the norm, the numerical radius and the spectral radius are respectively denoted by $\|\cdot\|, w(\cdot)$ and $r(\cdot)$. Let $\Sigma$ be a locally compact Hausdorff space and let $\mu$ be a regular Borel measure on $\Sigma$. Let $H$ be a direct integral of separable Hilbert spaces $H(s)(s \in \Sigma)$;

$$
\boldsymbol{H}=\int_{\Sigma} \bigoplus H(s) d \mu(s)
$$

Received by the editors April 8, 1970.

AMS 1969 subject classifications. Primary 4710, 4730; Secondary 4665.

Key words and phrases. Spectral radius, numerical radius, direct integral of operators, unilateral weighted shift, nilpotent, quasi-nilpotent.

1 The author is partially supported by Canadian NRC Grant 67-4058. 
and $A$, a direct integral of operators $A(s)$ on $H(s)$;

$$
A=\int_{\Sigma} \oplus A(s) d \mu(s) .
$$

For general theory of the direct integral, readers should confer with [1], or [3].

1. We shall denote three quantities by

$$
p=\mu \text {-ess. sup. }\|A(s)\| ; \quad w=\mu \text {-ess. sup. } w(A(s)) ;
$$

and

$$
r=\mu \text {-ess. sup. } r(A(s)) \text {. }
$$

THEOREM. If any two of the three quantities $p, w$ and $r$ are equal, then the spectral radius $r(A)$ of $A$ is equal to $w$.

The theorem will follow by a standard argument after establishing the following lemma.

Lemma. $w(A)=\mu$-ess. sup. $w(A(s)) \equiv w$.

Proof. Let $\phi=\int_{\Sigma} \bigoplus \phi(s) d \mu(s)$ be an arbitrary unit vector in $H$. Then, with the assumption $\phi(s) \neq 0$, (losing no generality)

$$
\begin{aligned}
|(A \phi, \phi)| & \leqq \int_{\Sigma}\left|\left(A(s) \frac{\phi(s)}{\|\phi(s)\|}, \frac{\phi(s)}{\|\phi(s)\|}\right)\right|\|\phi(s)\|^{2} d \mu \\
& \leqq \mu \text {-ess. sup. }\left|\left(A(s) \frac{\phi(s)}{\|\phi(s)\|}, \frac{\phi(s)}{\|\phi(s)\|}\right)\right| \\
& \leqq \mu \text {-ess. sup. } w(A(s)) \equiv w .
\end{aligned}
$$

Hence

$$
w(A)=\sup _{\|\phi\|=1}|(A \phi, \phi)| \leqq w .
$$

The lemma will be completed if we can prove that $w(A)$ is not strictly less than $w$. To this end, we let $\left\{\Lambda_{n}: n=0,1,2, \cdots, \aleph_{0}\right\}$ be the family of the disjoint dimension sets for the direct integral Hilbert spaces $H(s)$ so that for all $s$ in $\Lambda_{n}$, the Hilbert space $H(s)$ can be identified with a fixed Hilbert space $H_{n}$ (cf. [3]). Suppose that there were a constant $K$ satisfying $w(A)<K<w$. Then at least one of the dimension sets $\Lambda_{n}$ must contain a measurable subset $\tilde{S}$ with $0<\mu(\tilde{S})$ $<\infty$ such that, for some unit vector $x \in H_{n}$, the function $s \rightarrow(A(s) x, x)$ is measurable and $|(A(s) x, x)|>K$ for all $s$ in $\tilde{S}$. Since $\mu$ is a regular 
Borel measure, by Lusin's theorem, there is a compact subset $\hat{S}$ of positive measure contained in $\widetilde{S}$ such that the function $s \rightarrow(A(s) x, x)$ is continuous on $\hat{S}$. Let $\theta$ be the continuous function with the value in $[0,2 \pi]$ such that

$$
(A(s) x, x)=|(A(s) x, x)| e^{i \theta(s)} .
$$

It follows that there is a $\theta_{0}$ with $0 \leqq \theta_{0}<2 \pi$ satisfying $\operatorname{Re}\left(e^{i \theta_{0}} A(s) x, x\right)$ $>K$ on a subset $S$ of $\hat{S}$ of positive measure. Let $\phi$ be a vector field defined by

$$
\begin{aligned}
\phi(s) & =x, & & s \in S, \\
& =0, & & s \notin S .
\end{aligned}
$$

Then

$$
\begin{aligned}
|(A \phi, \phi)|^{2} & =\left|\left(e^{i \theta_{0}} A \phi, \phi\right)\right|^{2} \\
& =\left|\int_{S}\left(e^{i \theta_{0}} A(s) \phi(s), \phi(s)\right) d \mu\right|^{2} \\
& \geqq\left|\int_{S} \operatorname{Re}\left(e^{i \theta_{0}} A(s) \phi(s), \phi(s)\right) d \mu\right|^{2} \\
& >K^{2}(\mu(S))^{2}=K^{2}\|\phi\|^{2}>w(A)^{2}\|\phi\|^{2} .
\end{aligned}
$$

This contradiction to the definition of numerical radius establishes the lemma.

Proof OF THE THEOREM. We first observe that

$$
\mu \text {-ess. sup. }\left\|A(s)^{n}\right\|^{1 / n} \leqq\left(\mu \text {-ess. sup. }\left\|A(s)^{n}\right\|\right)^{1 / n}=\left(\left\|A^{n}\right\|^{1 / n}\right)
$$

because $\left\|A^{n}\right\|=\mu$-ess. sup. $\left\|A(s)^{n}\right\|$ for every integer $n$. Hence

$$
r=\mu \text {-ess. sup. } r(A(s)) \leqq r(A) \text {. }
$$

If $r=p$ or $r=w$, then the desired formula $r(A)=w$ follows immediately from the inequalities: $r \leqq r(A) \leqq w(A) \leqq w \leqq\|A\|=p$.

In case $w=p$, by the above lemma, $w(A)=\|A\|$. There exists a sequence of unit vectors $\left\{\phi_{n}\right\}$ such that $\left|\left(A \phi_{n}, \phi_{n}\right)\right|$ converges to $\|A\|$. Multiplying by a constant of modulus 1 if necessary, we may assume that $\left(A \phi_{n}, \phi_{n}\right)$ converges to $\|A\|$. But $\left|\left(A \phi_{n}, \phi_{n}\right)\right|$ $\leqq\left\|A \phi_{n}\right\| \leqq\|A\|$. This implies that $\|A\|$ is in the spectrum of $A$. Whence $r(A)=\|A\|$ and the proof of the theorem is complete.

2. Instead of working on the Halmos example directly, we consider a slightly generalized form of it:

EXAMPLE. Let $\Sigma$ be the positive half real line $[0, \infty)$, and $s \rightarrow H(s)$, 
a measurable field of Hilbert spaces such that, for each positive integer, $H(s)=E^{n}, s \in[n-1, n)$, where $E^{n}$ is the $n$-dimensional Euclidean space. If $f$ is a positive real-valued nondecreasing $\mu$ essentially bounded function, and if $\left\{\phi_{k}(s): k=1,2, \cdots, n(s)\right\}$ is an orthonormal basis for $H(s)$, then the operator field $s \rightarrow A(s)$ defined by

$$
\begin{aligned}
A(s) \phi_{k}(s) & =f(s) \phi_{k+1}(s), & k & =1,2, \cdots, n(s)-1, \\
& =0, & k & =n(s),
\end{aligned}
$$

is a field of nilpotent operators. But the direct integral

$$
A=\int_{\Sigma} \oplus A(s) d \mu(s)
$$

is not quasi-nilpotent.

In fact, $r(A)=\|A\|$. Let $\phi(s)$ be a unit vector in $H(s)$ with $\phi(s)$ $=\sum_{k=1}^{n(s)} \alpha_{k}(s) \phi_{k}(s)$, and $\Sigma_{k}\left|\alpha_{k}(s)\right|^{2}=1$. Then

$$
(A(s) \phi(s), \phi(s))=f(s) \sum \alpha_{k}(s) \bar{\alpha}_{k+1}(s) .
$$

Choose a vector $\phi(s)$ with $\alpha_{1}(s)=\alpha_{2}(s)=\cdots=\alpha_{n(s)}(s)=(n(s))^{-1 / 2}$. Then

$$
(A(s) \phi(s), \phi(s))=\frac{n(s)-1}{n(s)} f(s) \leqq w(A(s)) \leqq\|A\| .
$$

As $s$ increases, so do $n(s)$ and $f(s)$. Together with the fact that

$$
\|A\|=\mu \text {-ess. sup. }|f(s)| \text {, }
$$

we have $w=\|A\|$, which satisfies the hypothesis of the theorem. On the other hand, it is not hard to see that $r(A(s))<w(A(s))<\|A(s)\|$ for all $s \geqq 3$.

REMARK. In view of this example and our theorem, one might be tempted to conclude that if $r<w<p$, then $r(A)=r$. But this is not necessarily so, as shown in the following example:

Let $\left\{\alpha_{n}\right\}$ be a sequence defined by $\alpha_{1}=1, \alpha_{2}=\frac{1}{2}, \alpha_{3}=\alpha_{4}=\cdots=\frac{1}{3}$. Consider a weighted unilateral shift $A$ defined by the following sequence

$$
\alpha_{1}, 0, \alpha_{1}, \alpha_{2}, 0, \alpha_{1}, \alpha_{2}, \alpha_{3}, 0, \alpha_{1}, \alpha_{2}, \alpha_{3}, \alpha_{4}, 0, \ldots .
$$

There an easy calculation shows that $r(A)=\frac{1}{3}$. But $w(A)>\frac{1}{3}$. By our theorem, $w(A) \neq\|A\|$, because otherwise $r(A)=w(A)$. Hence we have just established a non-quasi-nilpotent which is a direct sum of nilpotents with $r<w<p$. 


\section{REFERENCES}

1. J. Dixmier, Les algèbres d'operateurs dans l'espace hilbertien (Algèbres de von Neumann), Cahiers Scientifiques, fasc. 25, Gauthier-Villars, Paris, 1957. MR 20 $\# 1234$.

2. P. Halmos, $A$ Hilbert space problem book, Van Nostrand, Princeton, N.J., 1967. MR 34 \#8178.

3. J. T. Schwartz, $W^{*}$-algebras, Gordon and Breach, New York, 1967. MR 38 \#547.

University of British Columbia, Vancouver, British Columbia 